The boy could walk independently and move both arms and legs without difficulties except for heelwalking on the left foot. There was mild left-sided weakness predominantly in the upper limb. He had exaggerated tendon reflexes in the left arm and leg and a left ankle clonus. The Babinski sign was bilaterally extensor. Reduced tactile gnosis in the left palm with compromised two-point discrimination and a reduced contralateral perception of hot and cold below the level of the lesion (indicating a Brown-Sequard syndrome) was detected. There was discrete muscular atrophy of the left lower limb.

CT and MRI of the upper spine showed pronounced compression of the spinal cord at the CII-CIII level by an invading exostosis (figs 1 and 2). Medial laminectomy of CII and CIII with extirpation of the exostosis as well as the posterior arches of $\mathrm{CII}$ and CIII was carried out in order to prevent the patient from developing tetraparesis. PAD showed a cartilaginous exostosis. Spasticity was still present two days after the operation but discrimination between hot and cold was normal and the extensor Babinsky sign of the right foot had disappeared. The tendon reflexes were exaggerated in the left arm but tactile gnosis was normal. The Babinski sign was still extensor in the left foot. One year after the operation left hand function was not fully restored and reduced muscle mass was noted. At present, two years after the operation, the boy has no significant disability but there is still slight recognisable left-sided weakness.

Multiple cartilaginous exostosis has variable expression. Eighty percent of cases have been diagnosed before the age of 10 years. The exostosis continues to grow until puberty and generally becomes apparent before the age of 30 . Occasionally neurological deficits appear and when the spine is involved the complications can be serious. A cervical location of the exostosis dominates among the reported cases..$^{3-5}$ We have found 17 published cases similar to the present one. The 15 who underwent surgery improved but only four recovered completely. ${ }^{6}$ The potential danger of the condition is illustrated by the fact that two patients died before operation. Altogether 14 patients had symptoms indicating spinal cord compression and 12 of these had symptoms from both the spinal cord and roots. The reported case serves to show that spinal cord compression may occur in hereditary multiple exostosis in adolescence and that early signs of spinal cord and root compression warrant a full radiological examination with the aim of performing surgery. CT is useful in revealing the origin

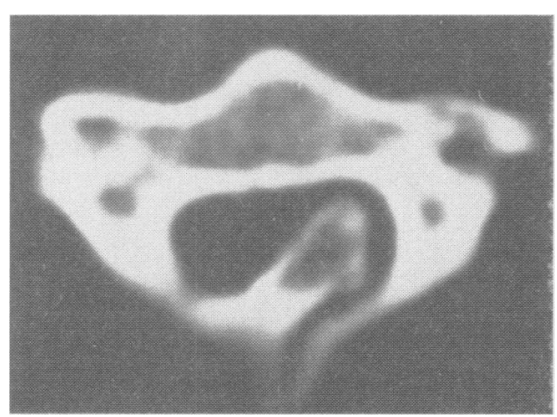

Figure $1 C T$ revealing the exostosis emerging from the left aspect of the inner vertebral arch (CII-CIII).

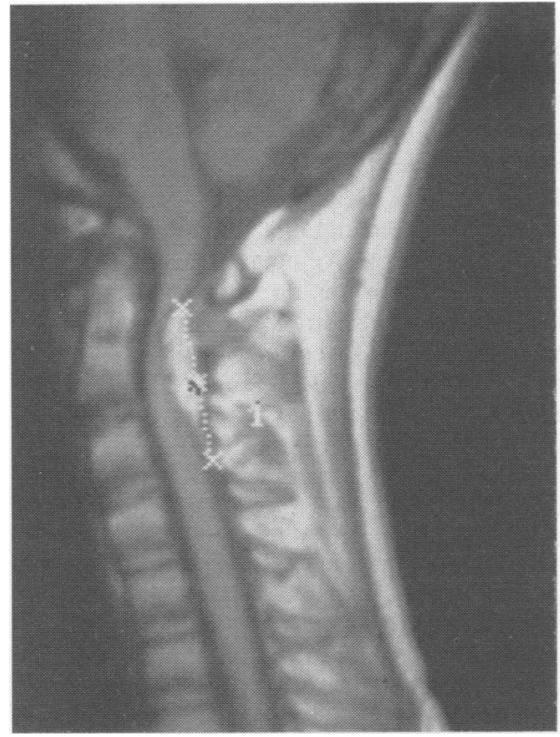

Figure 2 MRI depicting the spinal cord and the location of the compression.

and extent of the problem but is not optimal, whereas MRI is superior in visualising spinal cord compression.

I EMANUELSON Bräcke Östergård Regional Pediatric Habilitation Centre, Göteborg, Sweden

University of Göthenburg, Göteborg, Sweden Department of Pediatrics II A ROOS

Department of Neurosurgery

Correspondence to: Dr J Emanuelson, Bräcke Östergărd, Box 21062, 5-400 71 Göteborg, Sweden.

1 Chiurco AA. Multiple exostosis of bone with fatal spinal cord compression. Neurology 1970;20:275-8.

2 Madigan R, Worral T, McClain EJ. Cervical cord compression in hereditary multiple exostosis. Am $\mathcal{F}$ Bone foint Surg 1974; 56:401-4.

3 Tully RJ, Pickens J, Oro J, Levine C. Hereditary multiple exostosis and cervical Hereditary multiple exostosis and cervical Comput Assist Tomogr 1989;13:330-3.

4 Twersky J, Kassner G, Tenner MS, Camera Twersky J, Kassner G, Tenner MS, Camera
A. Vertebral and costal osteochondromas causing spinal cord compression. Am $\mathscr{F}$ Roentgenol Radium Ther Nucl Med 1975;1: 124-8.

5 Vinstein AL, Franken EA. Hereditary multiple exostosis. Report of a case with spinal cord compression. Am $f$ Roentgenol Radium Ther Nucl Med 1971;112:405-7.

6 Wen DY, Bergman TA, Haines SJ. Acute cervical myelopathy from hereditary multiple report Neurosurgery 1989;25:472-5.

\section{Bilateral tarsal tunnel syndrome}

A 52-year-old female school teacher with a positive history of hypertension presented with "sensation of heat" in distal parts of both soles for the past four months. This paraesthesia was accompanied in both feet by "electrical shocks" extending from the plantar arch to the tip of all toes and by occasional nocturnal numbness. No foot trauma, no precipitating factor, or relation to exertion or walking were reported. The patient had been helping herself by bathing her legs in cold water for 10 to 15 minutes, drying, and applying softening cream. This reportedly resulted in a complete relief of symptoms for about four hours.
On examination there were no signs of wasting or weakness of the small foot muscles. There was hypoaesthesia to pin-prick in the region of the medial and lateral plantar nerves. Tinel's sign was positive on the medial aspect of both ankles. General neurological state was normal. No abnormality of ankles and feet could be detected radiologically. Electrophysiological evaluation of both posterior tibial nerves showed a prolonged distal motor latency $(6.6 \mathrm{~ms})$ on the right and normal latency $(4.9 \mathrm{~ms})$ on the left side (normal distal motor latency range from the ankle to the abductor hallucis is $2 \cdot 9-5.3 \mathrm{~ms}$ ). There was no right medial plantar sensory action potential, whereas the amplitude of the left one was $0.3 \mu \mathrm{V}$ (normal $0.3-3.7 \mu \mathrm{V}$ ) and sensory conduction velocity was mildly decreased $(32 \mathrm{~m} / \mathrm{s}$; normal $35-48 \mathrm{~m} / \mathrm{s}$ ). Denervation activity in both abductor hallucis muscles was recorded by concentric needle electromyography. Thus clinical and neurophysiological findings indicated a diagnosis of bilateral tarsal tunnel syndrome.

Relief of symptoms was achieved after infiltration of $2 \%$ xylocaine behind the right medial malleolus.

The patient underwent two separate surgical procedures: firstly, an S-shaped incision was made behind the right medial malleolus to expose the posterior tibial nerve. The right posterior tibial nerve was compressed by an arcade of small branches of the tibial posterior artery, which were cut with bipolar coagulation. A $3 \mathrm{~cm}$ long segment of the posterior tibial nerve, thickened and firm on palpation, was separated from the epineurium under a microscope. Surgery on the other leg followed one week later when considerable relief of symptoms was confirmed on the operated leg. The left posterior tibial nerve was thickened and firm on palpation in a length of about $2 \mathrm{~cm}$. In a microscopic surgical procedure, epineurectomy, proximal and distal explorations were performed. No further abnormality that could have been the cause of the nerve compression was found. After both surgical procedures there was a complete relief of symptoms.

Tarsal tunnel syndrome characterised by entrapment of the posterior tibial nerve beneath the flexor retinaculum was first reported by Lam and Keck. ${ }^{12}$ Tarsal tunnel syndrome after an acute proximal process not affecting the ankle is rare. The role of ischaemia or traumatic proximal nerve damage causing greater susceptibility of the posterior tibial nerve has been discussed. ${ }^{34}$ Some other causes for tarsal tunnel syndrome have also been considered. ${ }^{5}$ To our knowledge, no other case of bilateral tarsal tunnel syndrome ha ms yet been reported. M DENIŠLIČ Institute of Clinical Neurophysiology, University Medical Centre, 61000 Ljubliana, Slovenia J BAJEC

Department of Plastic Surgery,
Ibn Sina Hospital, Kuwait

1 Lamm SJS. A tarsal tunnel syndrome. Lancet 1962;ii: 1354-5.

2 Keck C. The tarsal tunnel syndrome. 7 Bone foint Surg 1962;44A:180-2.

3 Augustijn P, Vanneste J. The tarsal tunnel syndrome after a proximal lesion. $尹$ Neurol Neurosurg Psychiatry 1992;55:65-7.

4 Fullerton MP. Effect of ischaemia on nerve conduction in carpal tunnel syndrome. F
Neurol Neurosurg Psychiatry 1963;26:385-97.

5 Poppi M, Giuliani G, Pozzati E, Acciarri N, Forti A. Tarsal tunnel syndrome secondary to intraneural ganglion. $\exists$ Neurol Neurosurg to intraneural ganglion. $\mathcal{f}$
Psychiatry 1989;52:1014-5. 